\title{
Intermittent ductal patency in healthy newborn infants: demonstration by colour Doppler flow mapping
}

\author{
M K Lim, K Hanretty, A B Houston, S Lilley, E P Murtagh
}

\begin{abstract}
Colour Doppler flow mapping was used to determine the time of closure of the arterial duct in 51 healthy newborn infants. Initial time of closure corresponded with previous reports: $20 \%$ on the first day, $82 \%$ by the second day, $96 \%$ by the third day, and $100 \%$ by the fourth day. Twenty infants were delivered by caesarean section and followed up for seven days even if the duct had apparently closed; in six intermittent patency was demonstrated with flow in the third, fourth or fifth day, although earlier functional closure had been observed. All were found to be closed on the sixth and seventh days. It is necessary to be aware of the phenomenon of intermittent closure in any study determining or assessing the effect of any intervention on ductal patency.
\end{abstract}

(Arch Dis Child 1992;67:1217-8)

Doppler echocardiography has demonstrated that in healthy term newborn infants functional closure of the arterial duct occurs later ${ }^{1-4}$ than previously had been reported. ${ }^{5}$ Colour Doppler flow mapping provides a sensitive and accurate tool to detect flow through a very small arterial duct $^{6}$ which might be missed by spectral Doppler echocardiography if an arduous and careful search is not carried out. ${ }^{7}$ We undertook a colour Doppler study of the timing of ductal closure in 1987 but did not report it as Reller et al did so before us. ${ }^{2}$ However, with colour Doppler techniques we observed intermittent ductal patency in the first week of life which has not been taken into account in other reports. ${ }^{1-4}$ As reported information on closure is becoming accepted as definitive, we considered it appropriate to report findings on ductal reopening. This should amplify and clarify the data on timing of ductal closure and ensure that misconceptions do not arise.

\section{Subjects and methods}

Fifty one newborn infants, born at term with a birth weight appropriate for gestational age, were studied. Thirty one were delivered by spontaneous vaginal delivery and 20 by lower segment caesarean section. There was no statistical difference in gestational age, sex, and birth weight between the two groups. All had normal Apgar scores at birth, and no clinical signs of cardiovascular abnormality or evidence of respiratory distress. Maternal consent was sought before the infant's inclusion in the study protocol. Newborn infants delivered by caesarean section stayed for at least seven days in the postnatal ward.

A Vingmed 700 system with a $5 \mathrm{MHz}$ transducer was used for the ultrasound studies. Standard high parasternal short axis and suprasternal views were obtained to attempt to image the arterial duct and to assess its patency with colour Doppler mapping.

All infants were studied within the first 24 hours of birth and subsequently at approximately 24 hourly intervals. The 31 delivered by vaginal delivery were studied only until ductal closure occurred. The 20 delivered by caesarean section remained in hospital longer and it was possible to study them daily for the first seven days of life.

\section{Results}

Studies were made each day and the cumulative total number of infants in whom initial ductal closure had occurred was observed as follows: first day (5-23 hours) in 20\% (10 of 51); second day (27-47 hours) in $82 \%$ (42 of 51); third day (51-71 hours) in 96\% (49 to 51 ); fourth day (75-91 hours) in $100 \%$ (all 51). The mode of delivery had no apparent effect on the timing of initial ductal closure $\left(\chi^{2}=2 \cdot 06 ; 0.5>p>0 \cdot 1\right)$.

Six of the 20 infants studied for seven days showed intermittent patency of the duct with flow again being demonstrated on the third, fourth, or fifth day, although initial functional closure had occurred (table). Colour Doppler maps showed that all the infants had closed ducts on the sixth day and they remained closed when observed for a further 24 hours.

Two small muscular trabecular ventricular septal defects were detected by Doppler scan. These infants did not have a murmur during their hospital stay (three and seven days). The

Intermittent patency of ductus arteriosus in healthy term newborn infants

\begin{tabular}{llllllll}
\hline Patient & \multicolumn{2}{l}{ Days of age } & & & & \\
No & 1 & 2 & 3 & 4 & 5 & 6 & 7 \\
\hline 1 & + & - & + & - & - & - & - \\
2 & - & - & + & - & - & - & - \\
3 & + & - & - & - & + & - & - \\
4 & + & - & + & + & - & - & - \\
5 & + & - & - & + & + & - & - \\
6 & + & + & - & + & - & - & - \\
\hline
\end{tabular}

+Ductal patency demonstrated by colour Doppler flow mapping; -no ductal patency demonstrated by colour Doppler flow mapping. 
infant discharged at three days developed one which is still present at 4 years of age while the other has never had a murmur although flow can still be demonstrated at 4 years of age.

\section{Discussion}

Sherman and Sahn noted that without colour flow mapping the Doppler detection of a small ductal jet can require an arduous search. ${ }^{7}$ Colour Doppler mapping simplifies the demonstration of ductal flow and is such a sensitive technique that in older patients we have found that Doppler demonstrates even a tiny arterial duct which cannot be recognised clinically. ${ }^{6}$ The observation of a ventricular septal defect which was inaudible in a child followed up to the age of 4 years adds further credence to the concept of minor cardiac defects which cannot be recognised by clinical examination. ${ }^{6}$ This may indicate that the incidence of congenital heart defects is higher than previously reported.

Our findings of timing of initial functional ductal closure are similar to previous reports using spectral or colour Doppler scans. ${ }^{1-4}$ Initial functional ductal closure occurred in the majority of patients by the second day of life and in all by the fourth. However, in previous reports observations were discontinued after initial ductal closure was noted. Our study has demonstrated reopening of the arterial duct in $30 \%$ of patients $(6 / 20)$ in whom patency was monitored for seven days. The mode of delivery was found not to affect the initial timing of ductal closure ${ }^{1}$ and this was confirmed in our patients. We have no reason to believe intermittent ductal patency should be confined to patients delivered by caesarean section.

The timing of normal ductal closure is of no clinical relevance in the normal infant. However, it may become important in studies assessing the effect of any intervention on ductal closure. Our observations suggests that final closure in newborn infants may occur later than previously reported. The possibility of intermittent ductal patency has not been monitored in previous reports but we consider that it is essential to take this into account in any future study which reports on the timing of ductal closure.

1 Gentile R, Stevenson G, Dooley T, Franklin D, Kawabori I, Pearlman A. Pulsed Doppler echocardiographic determination of time of closure in normal newborn infants. 于 Pediatr 1981;98:443-8.

2 Reller MD, Ziegler ML, Rice MJ, Solin RC, McDonald RW. Duration of ductal shunting in healthy preterm infants: an echocardiographic color flow Doppler study. f Pediatr 1988;112:441-6.

3 Drayton MR, Skidmore R. Ductus arteriosus blood flow during first 48 hours of life. Arch Dis Child 1987;62: during first.

4 Mandorla S. The ductus arteriosus in healthy newborn infants studied by continuous Doppler guided by twodimensional Doppler color echocardiography. G Ital Cardiol 1990;20:705-12.

5 Moss AJ, Emmanouilides GC, Duffie ER. Closure of the ductus arteriosus in the newborn infant. Pediatrics 1963;32:25.

6 Houston AB, Gnanapragasam JP, Lim MK, Doig WB, Coleman EN. Doppler ultrasound and silent ductus arteriosus. Br Heart $\mathcal{F} 1991$;6:97-9.

7 Sherman FS, Sahn DJ. Pediatric Doppler echocardiography 1987: major advances in technology. $\mathcal{F}$ Pediatr 1987; 